\title{
Microcontroller-Based Remote Temperature Monitoring System
}

\author{
Onawola Hazzan Jimmoh ${ }^{1}$, Sobowale Adedayo ${ }^{2}$, Obaje Samuel Enemakwu $^{3}$, \\ Iromini Nurudeen Ajibola ${ }^{4}$ \\ ${ }^{1,3,4}$ Computer Engineering Department, Federal Polytechnic, Offa, Kwara State \\ ${ }^{2}$ Computer Engineering Department, Federal Polytechnic, Ede, Osun State
}

\begin{abstract}
There is increase in death rate in hospitals due to inadequate attention to the patients, insufficient number of doctors as well as poor state of equipment make it difficult for the patients to receive proper and good medical treatment. This paper presents an efficient monitoring system, using microcontroller, which will monitor patient temperature remotely and send result to phones through Short Message Service (SMS) and Personal Computer (PC).The controller circuit was designed and interfaced to a sensor and thermistor and also interfaced inside with configured Single Inline Module (SIM) cards of phones to be connected. From the controller circuits, a system is interfaced with which would serve as Server, and from the server system, other system can be connected which would serve as Clients. The designed system was used to take temperature of different patients and it was ascertained that the result of our application gives accurate result when compared with clinical thermometer. With the new system developed, it will be very easy to measure patient temperature in order to reduce death rate being caused by diseases associated with increased temperature and provide platform for doctors to monitor the temperature of their patients thereby removing the barrier of distance.

Keywords: Single Inline Module (SIM) ,Short Message Service (SMS), Temperature, Remote Patient Monitoring, Internet, Personal Computer (PC), Thermistor, Server/Clients.
\end{abstract}

\section{Introduction}

Body temperature is the most vital sign among the most notable indexes of the human health, with the advantage of easy access. Moreover, unlike the X-ray, the measurement of body temperature has no effect on human health itself. There are some devices in the current market which can provide raw medical measurement data to patients and doctors, but the patients may not interpret the medical measurement into meaningful diagnosis since they have little medical background. On the other hand, if raw medical data is delivered to the doctor, it kills much time and may cause trouble, but in emergencies time can never be wasted. It is tough to share data over a large area within a short period. (Mohammad et al., 2013)

Remote health monitoring system has been an interesting topic recently among medical practitioners, engineers as well as IT professionals. However, the application of remote health monitoring system where doctor's can monitor patients' temperature via web and GSM is practically new in developing countries, Nigeria inclusive. The state of health especially temperature monitoring in these developing countries is faced with various challenges which ranges from high hospital bills, time and resources wastage, increased traffics in the hospital, and most painfully the increase in death rate due to inadequate prompt attention [1]. The insufficient number of doctors to patients in hospitals is another challenge faced in health monitoring, the poor state of equipment due to poor performance also contributes to the inadequacies of monitoring temperature.

Therefore, in order to address all the shortcomings in Remote Patient Monitoring (RPM) and inconveniences undergone by doctors and patients, the project was developed to automate the process of collection of patients' temperature and provide doctors the platform to remotely monitor their patients, removing the barrier of distance.

The central idea behind this research is to develop low cost intelligent remote monitoring system using mobile (cell phone) and internet. In past few years, there has been tremendous rise in number of mobile and internet users. Due to widespread growth of wireless cellular networks and drastic reduction in call rates and handsets, mobile usage has percolated all sections of society from business magnates to skilled and unskilled labourers. Cell phone and internet is gradually emerging as powerful tool for many health applications.

Remote Temperature Monitoring (RTM) has wide application in daily life. In modern day keeping an eye on temperature of places such as server rooms, hospital rooms, warehouses and green houses can help solve many problems. The use of normal temperature sensor, requires human physical presence to view the temperature readings, however with the development of GSM network, the temperature readings can be viewed anytime, anywhere. Through this technology, the temperature's readings is received via cell phones or other mobile devices, making distance a non issue[8]. 


\section{Related Works}

Remote Patient Monitoring (RPM) refers to a wide variety of technologies designed to manage and monitor a range of health conditions. Point-of-care monitoring devices - such as weight scales, glucometers, implantable cardioverter-defibrillators, blood pressure and temperature monitors which is our own concern in this project - may individually collect and report health data. They also may become part of a fully integrated health data collection, analysis and reporting system that communicates to multiple nodes of the health system. Such integrated systems provide alerts when health conditions decline, allowing patients, caregivers, and clinicians to intervene quickly and modify treatment plans as needed [1].

Just like RPM of temperature through GSM and internet, Reference [4] also used a Nokia N810 and the SHIMMER platform of wearable sensors to detect seizure events. [15] developed an integration of various sensors and systems in a network for comprehensive safety monitoring and smart home health care applications. AlarmNet is an example of such systems. It collects and analyzes various data streams to monitor a resident's overall wellness, known medical conditions, activities of daily living, and emergency situations. The whole project deals not only with wearable sensing technology but also with security/privacy issues in patient's data transfer, and real-time data streaming.

Reference [7] presented an algorithm for a minute-by-minute analysis of patients' activity level, based on data recorded using a single unit. The system was tested in 22 patients who were monitored over a period of 14 days. The authors also implemented a simple empirically-developed algorithm to determine if the subject was wearing the device thus providing a handle on compliance. Another interesting observation from the same study was that subjects tended to increase their activity level during the first few days of monitoring. This observation suggests that it is important that monitoring, if performed periodically, be performed over periods of time sufficient to avoid observing transitory effects introduced by the fact that the subject is aware of being monitored.

Movement sensors can also be used to automate clinical testing procedures. Reference [12] and [14] have proposed instrumented versions of the timed up-and-go test for identifying gait impairments due to PD (Parkinson's disease). They have shown that instrumented tests lead to an improved sensitivity to gait impairments compared to observation methods. Besides, sensor-based methods can also be extended to long term home monitoring. Based on this body of work, an ambulatory gait analysis system, based on wearable accelerometers, for patients with PD has been proposed by [12]

An ear worn sensor that can be used to monitor activities and levels of exertion in patients with chronic obstructive pulmonary disease was also developed [2]. Using sophisticated machine learning algorithms, the authors were able to identify several different types of physical activities and the intensity of those activities from a single ear worn sensor. Reference[3] measured human movement in three dimensions over 3 days and showed that the magnitude of the acceleration vector recorded in patients with chronic obstructive pulmonary disease was correlated with measures of patient's status such as the six-minute walk distance, the FEV1 (Forced Expiratory Volume in $1 \mathrm{sec}$ ), the severity of dyspnea, and the Physical Function domain of health-related quality of life scale.

A multi-sensor system, which measured galvanic skin response, heat flow and skin temperature in addition to motion, provided accurate estimates of energy expenditure was shown by [5] and [10]. Although not accurate at step counting, the multi-sensor system outperformed the step counters in estimating energy expenditure at slow walking speeds. With the development of wearable sensors and systems, which can be used for simultaneous monitoring of activities and several physiological parameters such as heart rate, respiration and oxygen saturation, it becomes possible to envision a more comprehensive status monitoring of patients with chronic obstructive pulmonary disease.

Reference [6] developed a remote monitoring system for analysis of sleep patterns in patients with early dementia. By performing objective monitoring of quality, quantity and rhythm of sleep the authors aimed to identify the level of cognitive impairment of individuals undergoing monitoring. The monitoring system included passive infrared (PIR) and bed pressure sensors. Preliminary results suggested that the sleep patterns of patients suffering from mild dementia are of lower quality when compared to control subjects. Other efforts to achieve the goal of assessing the progression of dementia have been made by other research groups.

Reference [13] designed a Wearable Kids' Health Monitoring System on Smartphone. In his project, a wearable device was developed. health-monitoring system for kids, which can log and communicate healthrelated data. The study started with reviewing current state of the art in health monitoring systems area. A questionnaire was designed and published to evaluate the main idea of the project and collect requirements of potential users. A doctor as a health professional was said to be interviewed during different steps of design and development of the system. The wearable device is constituted of 4 main parts: a TMP102 digital temperature sensor breakout from Sparkfun, a pulse sensor to measure heart rate, a Mini Pro Arduino microcontroller (3v), which collects and transmits data every 5 minutes, and a Bluetooth Mate Gold communication module from Sparkfun to send the data to the mobile application. 
An Automatic Patient's Heart Beat and Body Temperature Monitoring For Remote Doctor was designed by [11]. There they described how to monitor the heartbeat and the temperature of a patient continuously. The conventional practice has lots of disadvantages that, a nurse making rounds to check the pulse rates of the patients in hospital. For this, frequently it is necessary to disturb the patient. This project aims for the development of an electronic device, which monitor patient's heartbeat and temperature continuously. It will detect the abnormal changes in heartbeat and temperature and produce an alarm if supervision is necessary. The equipment design includes a receiver section connected with 2 light sensors to convert the biological response to electrical signal. The receiver section, through wireless connection receives the out put and produces alarm signal. The equipment was designed and tested in patients and found to be great success.

Reference [9] developed a device for Remote Monitoring of Heart Rate and Body Temperature. They built a portable device to provide a convenient solution for remote monitoring heart rate at the fingertip and body temperature using Ethernet technology and widely spreading internet. Most of the times in these cases, patients may not realize their actual conditions and even it is a common fact that there are no doctors by their side, especially in rural areas, but nowadays most of the diseases are curable if detected in time. They tried to make a system which may give information about one's physical condition and help him or her to detect these deadly but curable diseases.

\section{Methodology}

The qualitative research approach is employed in the realization of the objectives of this research paper. Computer application program and design were adopted to achieve the aims and objective of research work.

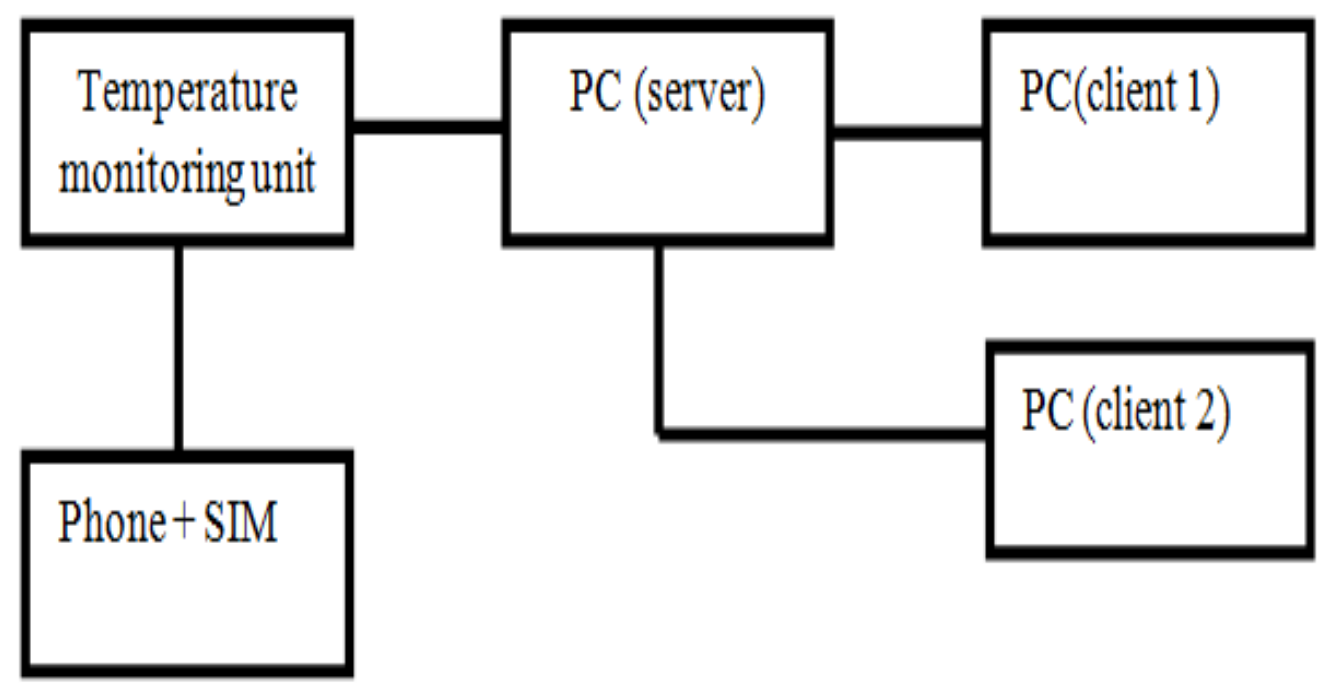

Fig. 1 Schematic Diagram of the Developed System

\section{A. Design and Implementation Approach}

The design and implementation approach defines the key components of the proposed system together with the interactions between these components. The flowchart representation of the developed model is shown in Figures 2.

The range of normal values is set before the system starts taking any readings. All the components required to take the readings of the temperature are initialised. The monitoring system now starts its operation in an unending loop until it is manually halted. The proposed system will read the temperature in analogue format then the Analogue-Digital-Converter (ADC) will convert the read values to digital format. The converted format will be compared to the preset values; if the read value is within the preset range the value will be transmitted to the local server where it will be displayed in tabular format and display results. If the read value is outside the range, an alert will be sent to the doctor, nurses, patient and patient' parents. 


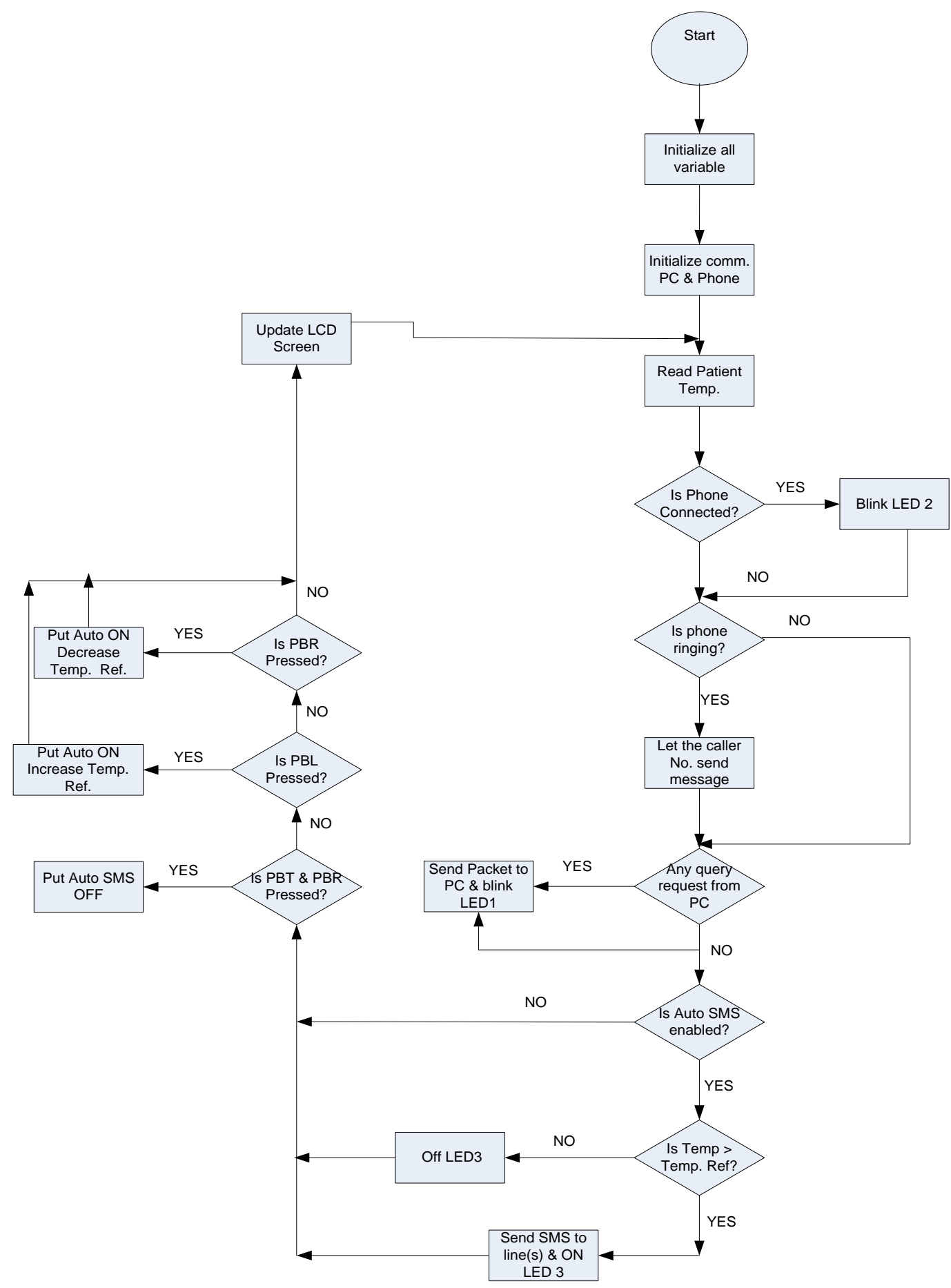

Fig. 2 Flowchart representation of the developed model

The Microcontroller is one of the major components of the proposed system; the proposed Clinical Decision Support System (CDSS) will be embedded into it. It will compare the measured values with the expected preset values and triggers the alarm ONLY when the measured values exceeds or is below the preset values. The PIC18F4550 is an 8-bit microcontroller, which has 10-bit Analog-to-Digital Converter (ADC). The temperature reading taken from the sensor attached to the wristwatch placed on the hand of the patient will be fed to an ADC port of the microcontroller in order to convert analog readings from the sensor to digital. Also, upon command, the microcontroller will read the temperature sample. It will then be converted and stored in the microcontroller memory as two 8-bit unsigned integers (0-255).

After completion of signals acquisition and comparison with preset values, the microcontroller then constructs the SMS messages as well as emails and packs the data samples in these messages to the desired length, it then communicates with the mobile phone using at-commands on its serial port to send the message(s). 
The device records temperature data continuously. When a temperature readings exceeds the preset values, an alarm is triggered and the doctor in charge is sent an email. Then, the measured values are sent to the local web server and displayed on the website of the hospital in a tabular format, this assists the doctor(s) in taking correct decisions based on the accurate data.

\section{System Testing And Results}

The results obtained were tested and confirmed under normal temperature and dustless environment and agreed with the expected result which were displayed on the Liquid Crystal Display (LCD). The results obtained were compared and tabulated as shown in table 1. From the result, it was seen that there was little or no variation in the patient's readings from the clinical thermometer and the monitoring unit.

Table 1 Tables showing Patients Temperature Readings

\begin{tabular}{|l|l|l|}
\hline Subject & Readings from Clinical Thermometer & Readings from patient body using Patient Monitoring Unit \\
\hline Patient A & 31 & 31.5 \\
\hline Patient B & 34.6 & 34.9 \\
\hline Patient C & 37.5 & 37.5 \\
\hline Patient D & 33.2 & 33.7 \\
\hline Patient E & 38.1 & 38.1 \\
\hline
\end{tabular}

\section{Conclusions}

We have presented remote temperature monitoring system using microcontroller to ease the work of doctors in hospitals. The designed system is capable of making work comfortable for the doctors in monitoring the temperature of their patients which has reduced death rate caused by diseases associated with temperature. The system is also appropriate for the monitoring of day-to-day activities in places like server rooms, hospital rooms, warehouses and green houses.

\section{References}

[1]. Alheraish, A. (October, 2004): "Embedded based remote monitoring of temperature", department of Mechanical and Industrial Engineering, University of Massachusetts.

[2]. Atallah L, Zhang J, Lo BPL, Shrikrishna D, Kelly JL, Jackson A, Polkey MI, Yang G- Z, Hopkinson NS (2010): "Validation of an ear worn sensor for activity monitoring in COPD",Am J Respir Crit Care Med 181,A1211.

[3]. Belza B, Steele BG, Hunziker J, Lakshminaryan S, Holt L, Buchner DM (2001): "Correlates of physical activity in chronic obstructive pulmonary disease", Nurs Res, Vol. 50, Pp. 195-202.

[4]. Dalton A, Patel S, Roy AC, Welsh M, Pang T, Schachter S, Olaighin G, Bonato P (2010) : "Detecting epileptic seizures using wearable sensor technologies", Book of Detecting epileptic seizures using wearable sensor technologies , Pp. 73-74

[5]. Furlanetto KC, Bisca GW, Oldemberg N, Sant'anna TJ, Morakami FK, Camillo CA, Cavalheri V, Hernandes NA, Probst VS, Ramos EM (2010): "Step counting and energy expenditure estimation in patients with chronic obstructive pulmonary disease and healthy elderly": accuracy of 2 motion sensors, Arch Phys Med Rehabil, Vol. 91,Pp. 261-267.

[6]. Haiying W, Huiru Z, Augusto JC, Martin S, Mulvenna M, Carswell W, Wallace J, Jeffers P, Taylor B, McSorley K (Dec 2010): "Monitoring and analysis of sleep pattern for people with early dementia, Bioinformatics and Biomedicine Workshops (BIBMW)", 2010 IEEE International Conference Vol. 18, Pp. 405-410.

[7]. Hecht A, Ma S, Porszasz J, Casaburi R (2009): " Methodology for using long-term accelerometry monitoring to describe daily activity patterns in COPD", Vol. 6, Pp. 121-129.

[8]. Meystre S., (2005): "The current state of telemonitoring" Telemed J E Health; 11(1):63-9,Department of Medical Informatics, University of Utah, Salt Lake City.

[9]. Mohammad Ashekur Rahman, Atanu Barai, Md. Asadul Islam, M.M.A Hashem ( 31 Mar 2013) : "Remote Monitoring of Heart Rate and Body Temperature", Journal reference: Procs. of the IEEE 2012 15th International Conference on Computer \& Information Technology (ICCIT 2012), Chittagong, Bangladesh, Pp. 411-416.

[10]. Patel SA, Benzo RP, Slivka WA, Sciurba FC (2007): "Activity monitoring and energy expenditure in COPD patients: a validation study", Vol. 4, Pp. 107-112.

[11]. Renjith p mathew, prasanna raja, manikandan ,karthik (May 2008): "Automatic patient's heart beat and body temperature monitoring for remote doctor", department of biotechnology, school of bioengineering, faculty of engineering and technology ,SRM university.

[12]. Salarian A, Horak FB, Zampieri C, Carlson-Kuhta P, Nutt JG, Aminian K (2010): "iTUG, a sensitive and reliable measure of mobility", IEEE Trans Neural Syst Rehabil Eng, Vol. 18, Pp. 303-310.

[13]. Shahram jalaliniya (2003): " A wearable kids' health monitoring system on Smartphone" IT University of Copenhagen.

[14]. Weiss A, Herman T, Plotnik M, Brozgol M, Maidan I, Giladi N, Gurevich T, Hausdorff JM (2010): "Can an accelerometer enhance the utility of the Timed Up \& Go Test when evaluating patients with Parkinson's disease?", Med Eng Phys Vol. 32, Pp.119-125.

[15]. Wood A, Stankovic J, Virone G, Selavo L, He Z, Cao Q, Doan T, Wu Y, Fang L, Stoleru R (2008): "Context-aware wireless sensor networks for assisted-living and residential monitoring" ,IEEE Network Vol. 22, Pp. 26-33 